\title{
Design principles for high quality electron beams via colliding pulses in laser plasma accelerators
}

\author{
E. Cormier-Michel, ${ }^{*}$ V. H. Ranjbar, ${ }^{\dagger}$ D. L. Bruhwiler, ${ }^{*}$ and J. R. Cary \\ Tech-X Corporation, 5621 Arapahoe Avenue, Boulder, Colorado 80303, USA
}

M. Chen, ${ }^{\S}$ C. G. R. Geddes, G. R. Plateau, ${ }^{\|}$N. H. Matlis, and W. P. Leemans

BELLA Center, Lawrence Berkeley National Laboratory, 1 Cyclotron Road, Berkeley, California 94720, USA

(Received 22 October 2013; revised manuscript received 5 August 2014; published 5 September 2014)

\begin{abstract}
Laser plasma based accelerators have the potential to reduce dramatically the size and cost of future particle colliders and light sources. Production of high quality beams along with reproducibility, tunability, and efficiency are required for many applications. We present design principles for two-pulse colliding laser pulse injection mechanisms, which can meet these requirements. Simulations are used to determine the best conditions for the production of high quality beams: high charge, low energy spread, and low emittance. Simulations also allow access to the internal dynamics of the interaction, providing insight regarding further improvement of the beam quality. We find that a $20 \mathrm{pC}$ beam can be accelerated to $300 \mathrm{MeV}$ in $4 \mathrm{~mm}$ with only a few percent energy spread and transverse normalized emittance close to $1 \mathrm{~mm}$ mrad, using a $10 \mathrm{TW}$ laser. We demonstrate that this design scales according to linear theory. Control of the laser pulse mode content and subsequent evolution in the plasma channel are shown to be critical for achieving the highest beam quality.
\end{abstract}

DOI: 10.1103/PhysRevSTAB.17.091301

PACS numbers: 52.38.Kd, 41.75.Jv, 52.65.Rr

\section{INTRODUCTION}

Laser plasma based accelerators (LPAs) are able to sustain accelerating gradient 3 orders of magnitude higher than conventional accelerators, making them attractive for the next generation of particle accelerators [1]. Applications such as high energy physics or production of light sources (gamma-ray and x-ray sources), require tunable, reproducible high quality electron beams, i.e., low energy spread and low emittance beams, while maximizing energy transfer between the laser and the electrons. Production of high quality electron beams in LPA was first demonstrated at the $100 \mathrm{MeV}$ level [2-4] using $10 \mathrm{TW}$ class lasers, and later on at the $0.5-1 \mathrm{GeV}$ level [5], where longer interaction distance and higher laser power $(\sim 40 \mathrm{TW})$ were used. In these experiments, the beam production relied on the self-trapping of background plasma electrons. Because the self-trapping process is highly nonlinear, the properties of the beam are

\footnotetext{
*estelle.cormier@gmail.com

Presently at Brookhaven National Laboratory, Upton, NY 11973, USA.

Presently at RadiaSoft LLC, Boulder, CO 80304, USA.

${ }^{\S}$ Presently at Shanghai Jiao Tong University, Shanghai, 200240, China.

"Presently at UCLA, Department of Physics and Astronomy, Los Angeles, CA 90064, USA.

Published by the American Physical Society under the terms of the Creative Commons Attribution 3.0 License. Further distribution of this work must maintain attribution to the author $(s)$ and the published article's title, journal citation, and DOI.
}

difficult to reproduce at every shot, even though some parameter regimes have been reported where reproducible electron beams have been obtained [5,6]. Several techniques are currently being explored to improve reproducibility and tunability of electron beams produced by LPA such as injection in negative plasma density gradient $[7,8]$, injection via ionization of inner electrons of a high $Z$ atom [9-14], and colliding pulse injection (CPI) $[15,16]$. Those three methods have in common that the injection of the electron beam can be controlled separately from the acceleration. For high energy beams, experiments [3,5], theory, and simulations [1] show it is important to combine injection control with plasma guiding of the laser to extend the focused propagation length.

The original CPI method uses three laser pulses [15]: a main, high intensity, laser pulse which drives a high gradient wakefield and two low intensity injection pulses orthogonally polarized to the main laser. One of the injection pulses follows the main driver pulse, while the second injection pulse propagates in the opposite direction. When the two injection pulses collide, they create a slow beat wave able to trap electrons from the background plasma. As electrons are trapped in the slow beat wave they get a kick in momentum and phase which is able to position them on the trapped orbits of the main wakefield where they are accelerated to high energies. This mechanism can create short, isolated, high quality electron bunches [17]. The same scheme can be used with two laser pulses [18-20], where the main driver also acts as the forward 
going injection pulse. This is simpler but yields less control over the beam phase. This injection process does not rely on previous self-evolution of the lasers, improving control. The properties of the electron beam can be tuned by changing laser and plasma parameters.

The beat wave, i.e., the point of collision between the two laser pulses, should overlap the accelerating and focusing region of the wakefield driven by the main laser pulse, which, in the two-pulse scheme, is located between the phases $k_{p} \zeta=-3 \pi / 2$ and $k_{p} \zeta=-2 \pi$ behind the leading edge of the laser pulse, where $k_{p}=2 \pi / \lambda_{p}$, and $\lambda_{p}=$ $\sqrt{m c^{2} / 4 \pi n_{0} e^{2}}$ is the plasma wavelength, with $n_{0}$ the plasma density, and $\zeta=x-c t$. The driver pulse should also be long enough to overlap with this region. Typically the characteristic pulse length $L_{0} \sim \lambda_{p} / 2$, i.e., extending back to the phase $\sim-\pi$, is an optimum choice for trapping as it is reasonably close to the resonant condition for wakefield generation, yielding a large amplitude wakefield, while also ensuring that the driver overlaps the accelerating phase of the wake. Trapping will occur when the top of the separatrix of the beat wave exceeds the bottom of the separatrix of the wakefield and when the bottom of the separatrix of the beat wave is lower than the plasma fluid electron momentum. This gives a minimum value for the product $a_{0} a_{1}$ for the trapping threshold [18], and typically for $a_{0} \sim 1, a_{1} \gtrsim 0.1$.

Triggered injection via the two-pulse CPI method has been been demonstrated experimentally $[16,21]$ and experiments are continuing to improve electron beam quality and energy. For example, experiments are currently taking place at LOASIS program at LBNL, using their $10 \mathrm{TW}$ Ti: sapphire laser system [22]. A $0.6 \mathrm{~J}, 50$ fs $(15 \mu \mathrm{m})$ FWHM laser pulse is used to drive the wakefield. The injection is triggered by colliding the main laser with a second $\lesssim 0.25 \mathrm{~J}, 100$ fs $(30 \mu \mathrm{m})$ laser at a $19^{\circ}$ angle inside a hydrogen gas jet. The laser pulse can be expressed in terms of the normalized vector potential such that $a=e A / m \omega^{2} \sim a_{0,1} \exp \left(-x^{2} / L_{0,1}^{2}\right) \exp \left(-r^{2} / w_{0,1}^{2}\right)$, where $a_{0,1}=0.85 \times 10^{9} \lambda[\mu \mathrm{m}] \sqrt{I_{0,1}\left[\mathrm{~W} / \mathrm{cm}^{2}\right]}$, and $\lambda$ is the laser wavelength and $I$ the laser peak intensity. The indices 0,1 refer to the main pulse and the lower energy collider pulse, respectively. This system was used to produce $\sim 100 \mathrm{MeV}$ monoenergetic electron beams [3] using the self-injection scheme. Similar energy lasers are now available in more compact form, enabling production of high quality beams for tabletop light sources [23].

Simulations have been used to develop understanding of the colliding pulse mechanism using test particle codes to verify trapping thresholds and condition for production of high quality beams $[15,17,18]$. The use of fully selfconsistent particle-in-cell (PIC) codes is sometimes necessary to completely encompass the full physical phenomena [24], and allow us to understand the internal dynamics of the interaction [25], in order to better explain experimental results [26,27] and better design the injection process $[19,28]$. In previous simulations, mainly dependence of laser pulse intensities, laser pulse polarizations [29], and location of the collision along the drive laser propagation axis on the electron beam properties were studied. For efficient, high quality accelerators, further self-consistent simulations are needed to understand colliding pulse injectors with plasma channel guiding and the importance of laser evolution, tuning of the injector to increase beam performance per laser joule, the scaling of the mechanism to produce desired beam energy and charge, and the impact of non-Gaussian laser modes typical of experiments.

In this paper self-consistent VORPAL [30] simulations are used to show that use of plasma channel guiding can significantly improve performance and efficiency of colliding pulse injected LPAs. Control of laser focusing in uniform and channeled plasmas is important to control injected bunch phasing and to maintain injector efficiency. The effects of non-Gaussian laser modes representative of typical experiments on injection and acceleration are characterized. Scaling of the injector and accelerator performance is demonstrated to allow design for applications requiring different charges and energies. The channel guided performance is contrasted with unchanneled conditions typical of current experiments. Parameter scans are performed to guide the experiments on the production of high quality electron beams using CPI. Comparison with experimental data helps to validate simulations results. We show with the simulations that the properties of the trapped electron beam can be tuned by changing laser or plasma parameters. These simulations show that the colliding pulse injection scheme can use more efficiently the laser energy as it does not require prior propagation of the laser into the plasma to induce trapping, and electron beams close to $0.5 \mathrm{GeV}$ can be produced using the $10 \mathrm{TW}$ laser system. This makes it more affordable to produce beams relevant to light source applications, including gamma-ray sources using Compton scattering [31].

The remainder of this paper is organized as follows. In Sec. II we show that 2D simulations capture the essential physics seen in 3D; in Sec. III we perform density scans and evaluate tunability of the injector; Sec. IV shows the effect of the laser driver focusing on the final beam quality; in Sec. V parameter scans are performed to characterize the injection as a function of delay, plasma density, and laser amplitude and simulation results are compared with experimental data. Finally, in Sec. VI we evaluate the effects of higher order mode components in the laser driver on the final beam quality.

\section{COMPARISON BETWEEN 3D AND 2D SIMULATIONS}

Optimization of the injector and accelerator performance requires many simulations, while $3 \mathrm{D}$ runs are still computationally too expensive to be done in large numbers. 
Reduced models such as envelope models or boosted frame simulations, which can greatly reduce computational effort for calculations of acceleration, are not practical solutions for colliding pulse. This is because modeling the beat wave requires resolving the laser wavelength and polarization for both forward and backward propagating waves. A thorough parameter scan is therefore only practical using $2 \mathrm{D}$ simulations. Previous simulations of LPA in the nonlinear regime showed that 2D cannot always capture all the physics, due to self-modulation of the laser pulse [27,32]. Here we therefore characterize how well 2D simulations represent 3D results when modeling CPI at parameters of the present study. Future solutions may include simulating trapping with full PIC and transition to a reduced model once the beam has been trapped, which would require careful reinterpolation between the different computational grids during transition.

To allow sensitive evaluation of dimensional effects, simulations were conducted in $2 \mathrm{D}$ and $3 \mathrm{D}$, for laser and plasma parameters which previous works $[18,33]$ show to produce colliding pulse injection of a high quality electron beam. Laser parameters were $a_{0}=2, L_{0}=10 \mu \mathrm{m}, w_{0}=$ $6 \mu \mathrm{m}$ and $a_{1}=0.5, L_{1}=18.15 \mu \mathrm{m}, w_{1}=11 \mu \mathrm{m}$. Density on axis was $n_{0}=4.2 \times 10^{18} \mathrm{~cm}^{-3}, \lambda_{p}=16 \mu \mathrm{m}$. The simulation grid size is $d x=\lambda_{0} / 36$ in the longitudinal direction for the 3D simulation and $d x=\lambda_{0} / 48$ in the 2D simulation, and $d y=d z=\lambda_{0} / 3$ in the transverse direction. Here, $\lambda_{0}=0.8 \mu \mathrm{m}$ is the wavelength of both laser pulses. Given the laser parameters, the laser power is not quite the critical power for self-focusing: $P / P_{c} \simeq 0.7$, where $P_{c}[\mathrm{GW}]=17.4\left(\lambda_{p} / \lambda_{0}\right)^{2}$ is the critical power. Hence, in a uniform plasma profile, the laser diffracts, leading to a decrease of intensity with propagation distance and a decrease of the accelerating gradient for the trapped electron bunch. A plasma channel with transverse profile of the form $n(r)=n_{0}+\Delta n_{c}\left(r / w_{M}\right)^{2}$, with $\Delta n_{c}\left[\mathrm{~cm}^{-3}\right]=$ $1.13 \times 10^{20} / w_{M}^{2}[\mu \mathrm{m}]$ and $w_{M}$ the matched channel radius [1], needs to be introduced to keep the high intensity of the laser over many Rayleigh lengths. However, the matched condition $w_{M}=w_{0}$, needs to be relaxed to compensate for the self-focusing of the laser pulse in the channel [34]: $w_{M}=9.2 \mu \mathrm{m}$ is used in this case.

The trapped electron beam parameters at dephasing are similar for both simulations, as seen in Fig. 1. The beam loading physics however is different in $2 \mathrm{D}$ and $3 \mathrm{D}$. The fields appear to be more beam loaded in 3D, consistent with previous work [35], leading to about $20 \%$ less energy gain, the beam peak mean energy being $280 \mathrm{MeV}$ in 3D and $367 \mathrm{MeV}$ in 2D, with an energy spread of $2 \%$ in both cases. In $3 \mathrm{D}$, the transverse normalized emittance is $3.6 \mathrm{~mm}$ mrad in the plane of the laser polarization, and $0.7 \mathrm{~mm} \mathrm{mrad}$ in the direction orthogonal to the laser polarization. For comparison in 2D, we performed two different runs, one where the laser is polarized in the plane of the simulation and one where the laser is polarized outside the plane of the simulation. The transverse normalized emittance in the
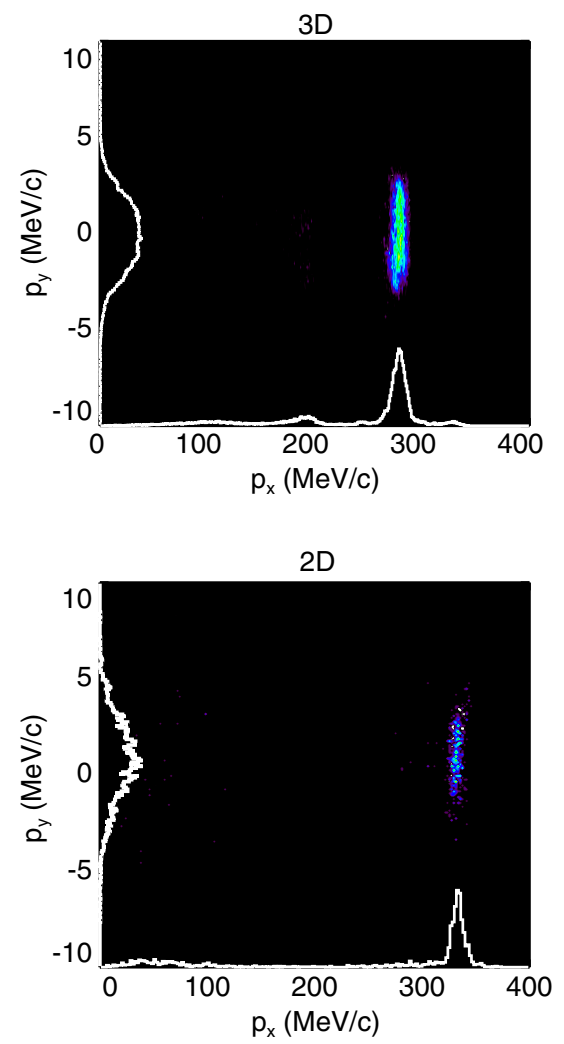

FIG. 1. Longitudinal and transverse momentum of the trapped electron beam at dephasing for $3 \mathrm{D}$ and $2 \mathrm{D}$ simulations. The plot of the 3D simulation appears more dense because of the higher number of total macroparticles in the simulation, but numbers for energy spread and emittance are similar in both cases.

plane of the simulation is $1.7 \mathrm{~mm}$ mrad and $0.7 \mathrm{~mm} \mathrm{mrad}$, respectively. Note that the emittance of the beam might not be converged even for the highest resolution, as suggested by Table I of Appendix C, which could explain the modest discrepancy between 3D and 2D. It has been shown previously that the emittance is very sensitive to numerical noise and requires stringent numerical parameters to be converged [32], and work by several groups is in progress to improve convergence. This could also explain the larger emittance in the plane of polarization of the laser pulse, although we have not studied convergence of the emittance with resolution for the emittance outside the polarization plane. While overlapping of the electron beam with the driver laser pulse as the electron beam dephases could lead to greater numerical noise, it has also been shown that this could lead to larger emittance in the direction of polarization [36]. In general, this can be overcome by using the 3 -pulse scheme, where the electron bunch would overlap with a laser of much lower intensity, reducing the transverse momentum spread. The charge of the beam in $3 \mathrm{D}$ is about 20 pC. In 2D, one can only obtain a charge per unit of length. We observe that a good estimate of the trapped charge, similar to what is observed in $3 \mathrm{D}$, can be calculated 
by multiplying the $2 \mathrm{D}$ charge by the spot size of the laser driver.

Reducing the collider amplitude in 3D simulations confirms that charge and energy are controllable in this scheme. By reducing the value of $a_{1}$ to 0.4 , the trapped charge is reduced to $17 \mathrm{pC}$ with an increase of the final energy to $300 \mathrm{MeV}$ and 2\% energy spread. This confirms the trend observed in the more detailed parameter scans performed in 2D in Sec. V (Fig. 8). We do not observe a significant change in the beam emittance, compared to the case where $a_{1}=0.5$, either in or out of the plane of the driver pulse polarization.

In the cases above, the lasers were polarized in the same plane as the plane where the collider is intersected with the driver pulse (i.e., polarized in the $y$ direction). The results are similar (with a few percent difference) when the lasers are polarized in the opposite direction (i.e., in the $z$ direction). All of the cases here use like polarization for both beams, and the difference is due to the (small) crossing angle of the two beams. Crossed polarization has been previously described in collinear geometry by the authors of [26].

For the parameters of the present study, operating in the mildly nonlinear wake regime and with trapping triggered by colliding pulses, comparisons of $2 \mathrm{D}$ and $3 \mathrm{D}$ simulations shown above indicate that the trapping and acceleration process is reasonably accurately modeled in $2 \mathrm{D}$, albeit with $\sim 20 \%$ differences in energy gain, which in this case is primarily due to the differences in beam loading. This allows parameter scans to be conducted in $2 \mathrm{D}$ to characterize injector performance. As shown above, these were checked at specific points using 3D simulations with careful choice of parameters based on the 2D results. The validity of $2 \mathrm{D}$ results in this regime is consistent with previous results [37] that showed that 2D was representative for the wake and particle acceleration in mildly nonlinear cases without significant self-focusing induced changes in the laser spot size and before significant laser depletion (as is the case in the present study). Similarly, the colliding pulse particle injection effect is primarily a 1D effect $[15,17,24]$ and hence is well captured by 2D simulations. The ability to conduct 2D simulations must be verified for each regime of interest. For example when laser depletion is important it will be stronger in $3 \mathrm{D}$ which will affect results [37]. Similarly the physics of injection during highly nonlinear self-trapping, where the wakefield can depend on prior laser evolution and trapping, is strongly dependent on the physics at the back of the bubble, and cannot be well be represented in 2D [27,32].

\section{INDEPENDENT TUNING OF BEAM ENERGY VIA PLASMA DENSITY}

Two-dimensional parameter scans are performed to characterize the behavior of the injector. As above we use a preformed plasma channel, which allows guiding of driver pulses with power below the self-guiding threshold and improves the beam properties including increasing energy gain $[1,3,5]$.

While typical experiments scan one variable while keeping others constant, simulations have shown [35,37] that accelerator performance can be predicted by linear scaling laws even for cases not directly soluble by the theory when the dimensions of the problem are kept constant compared to the plasma wavelength, and the normalized laser intensity is constant. Here we characterize scaling of the injector. We then illustrate how the injector performance can be controlled about this scalable design by changing the plasma density alone. Other studies have also characterized single parameter scans including laser intensity scans [17-19,24].

First, the plasma density is varied along with the dimensions of the laser pulse, so that the laser dimensions stay proportional to $\lambda_{p} \propto 1 / \sqrt{n_{0}}$, i.e., $L / \lambda_{p}$ and $w_{0} / \lambda_{p}$ are kept constant, starting from the laser parameters described in Sec. II, at a density of $4.2 \times 10^{18} \mathrm{~cm}^{-3}$. The laser is guided by a plasma channel matched to a spot size scaling with the change in density such that $w_{M} / w_{0}=1.5$. We observe that the properties of the beam follow remarkably well the scalings from the linear theory [1]. Figure 2 shows the evolution of dephasing length, beam charge, and beam energy. The dephasing length scale evolves as predicted by theory, i.e., $L_{d}=\lambda_{p}^{3} / 2 \lambda_{0}^{2}$, with $\lambda_{0}$ the laser wavelength, which is represented by the dashed line on Fig. 2. The energy scales as $1 / n_{0}$, which is expected when multiplying the dephasing length by the amplitude of the accelerating field, which scales as $I / n_{0}$. The 3D charge scales as $1 / \sqrt{n_{0}}$, which is proportional to the interaction volume of the two lasers times the density of the plasma. This scaling is similar to the beam loading scaling law [35], and indeed we observe the wake to be loaded by the same amount for all densities. Energy spread remains close to $2 \%$ and the

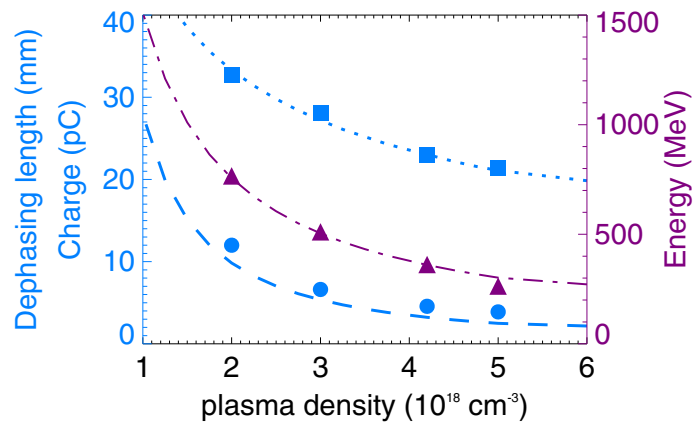

FIG. 2. Variation of dephasing length (blue circles, left axis), trapped charge (blue squares, left axis) and beam energy (purple triangles, right axis) as a function of plasma density when the laser dimensions scale proportionally to the plasma wavelength. The blue dashed line shows the theoretical dephasing length $L_{d} \sim \lambda_{p}^{3} / 2 \lambda_{0}^{2} \propto 1 / n_{0}^{3 / 2}$, the blue dotted line shows the scaling of the charge corresponding to the same amount of beam loading $Q \propto 1 / n_{0}^{1 / 2}$ and the purple dash-dotted line shows the scaling for the energy gain $E \propto 1 / n_{0}$. 
emittance at $2.2 \mathrm{~mm} \mathrm{mrad} \pm 0.8 \mathrm{~mm}$ mrad. Note that the simulations are done with the lasers polarized in the plane of the 2D simulation; we can expect that out of the plane, the emittance of the beam would be below $1 \mathrm{~mm}$ mrad. These scaling laws allow quick design of the injector according to the required accelerator performance. For example, current experimental parameters can produce $300 \mathrm{MeV}$ beams, which is suitable for application such as $1.7 \mathrm{MeV}$ gamma-ray sources. $\mathrm{GeV}$ beams can be produced with a plasma at $n_{0}=10^{18} \mathrm{~cm}^{-3}$ with $1.4 \mathrm{~J}$ and $0.5 \mathrm{~J}$ driver and collider laser pulses, respectively.

Current experiments allow change of the plasma density while the laser parameters stay constant. Simulating this case, with laser parameters $L_{0}=9.7 \mu \mathrm{m}, w_{0}=6.14 \mu \mathrm{m}$, $a_{0}=2$ for the drive pulse and $L_{1}=18.15 \mu \mathrm{m}, w_{1}=11 \mu \mathrm{m}$, $a_{1}=0.5$ for the collider, we observe a plateau in the trapped charge $(Q=18.9 \pm 1.3 \mathrm{pC})$ between $n_{0}=3$ and $6 \times 10^{18} \mathrm{~cm}^{-3}$, as seen in Fig. 3. This corresponds to a range in density where the resonant condition, $L_{0}=\lambda_{p} / \pi$, is met, balancing overlap between the laser pulse and the phase in the wake where particles can be trapped, while still driving a wakefield with strong enough amplitude such that the particles of the background plasma can be kicked on the trapped orbits by the beat wave. All the parameters of the beam stay constant within this density range, with $\Delta E / E=2.1 \pm 0.2 \%, \quad \epsilon_{n}=1.76 \pm 0.16 \mathrm{~mm} \mathrm{mrad}$, beam radius $=0.5 \pm 0.03 \mu \mathrm{m}$. Only the energy gain decreases as the density increases; this is due to the change in dephasing length as the density varies. This means that the energy of the beam can be tuned independently of other
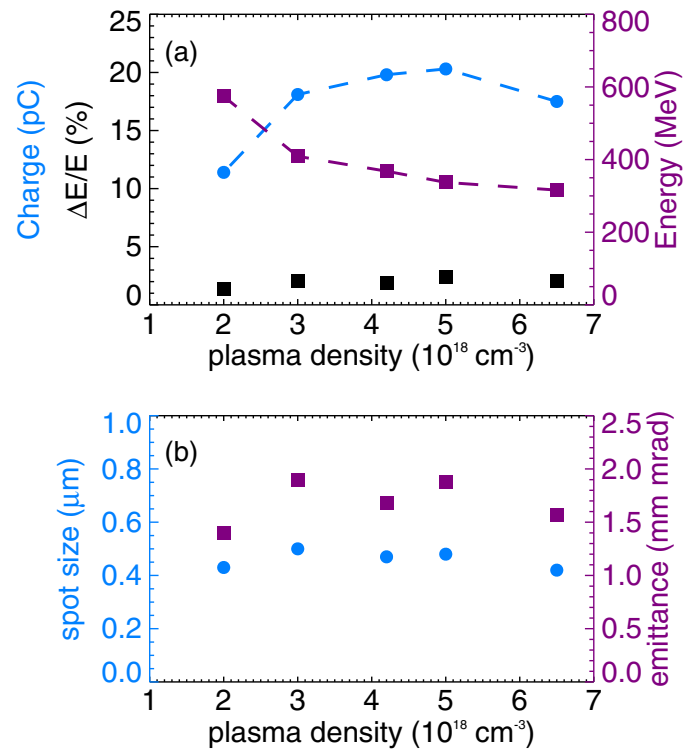

FIG. 3. (a) Variation of the trapped charge (blue circles, left axis), energy (purple squares, right axis), and rms energy spread (black squares, left axis) as a function of the plasma density when the laser dimensions are kept constant. (b) Variation of the electron beam rms radius (blue circles, left axis) and emittance (purple squares, right axis) as a function of plasma density. beam parameters, including beam charge and energy spread, by only changing the plasma density. Related work [16] has shown that energy can also be tuned by varying the collision position, but in that case energy spread and charge varied more strongly with energy, which has been attributed [27] to nonlinear self-focusing of the laser for later collision positions.

\section{CONTROL OF LASER PULSE EVOLUTION TO ACHIEVE HIGH QUALITY ELECTRON BEAMS}

The slow beat wave created by the collision of the two counter-propagating laser pulses allows electrons of the background plasma to be positioned on the trapped and focusing orbits of the wakefield [17]. However, if the laser pulse evolves during or after the interaction, e.g., due to mismatch of the laser pulse in the plasma channel, those orbits can be displaced in phase-space, and some of the initially trapped electrons are no longer positioned on a trapped and focusing orbit and are lost from the accelerating bunch. The interaction of colliding pulses with wake phasing evolution driven by laser focusing $[8,32,38]$ must therefore be controlled to achieve efficient injection.

Figure 4 shows that self-focusing of the laser pulse in the plasma channel can be detrimental for the trapped electron
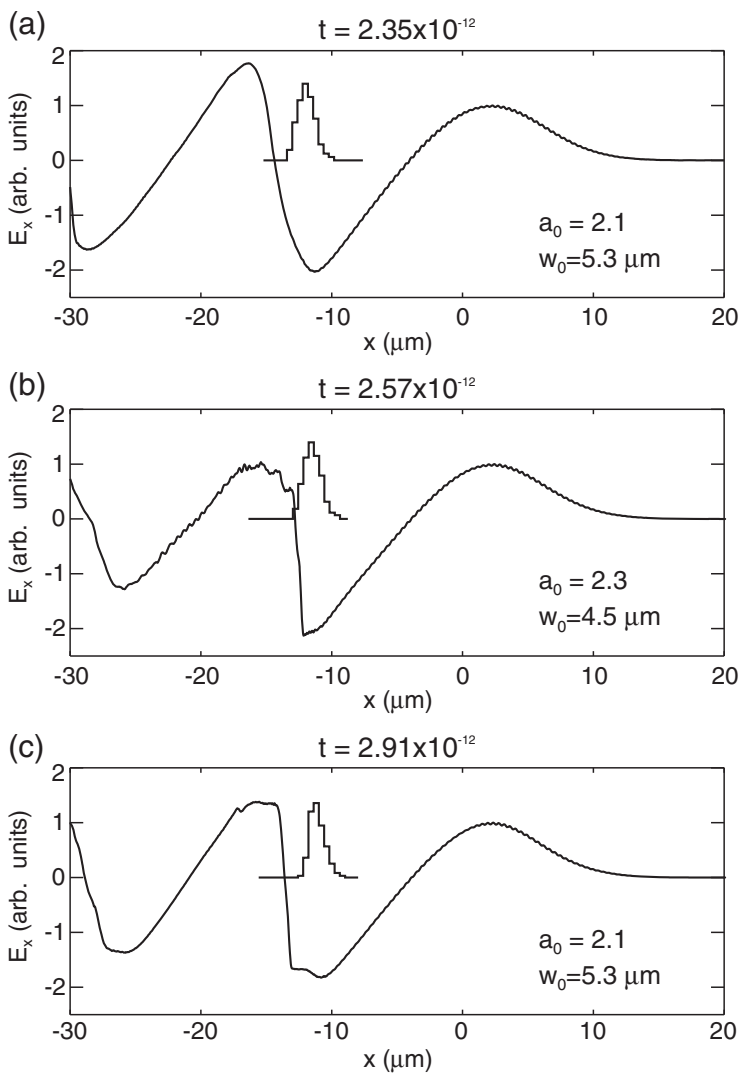

FIG. 4. Longitudinal electric field (on axis) at different times and histogram density (integrated over the transverse dimension) of the trapped electron beam. 
beam. Figures 4(a)-(c) show the longitudinal electric field on axis and the position of the trapped electron beam in sequential times after injection. Laser parameters are $a_{0}=2, L_{0}=10 \mu \mathrm{m}, w_{0}=6 \mu \mathrm{m}$ and plasma density on axis $n_{0}=4.2 \times 10^{18} \mathrm{~cm}^{-3}$. The longitudinal electric field is normalized so its first peak on the right is at the same amplitude. In each frame is also specified the laser intensity $\left(a_{0}\right)$ and spot size $\left(w_{0}\right)$ at this particular point in time. The pulse is propagating in a plasma channel of matched spot $w_{M}=7.3 \mu \mathrm{m}$ and is focused at the left edge of the plasma. The laser is not perfectly matched and so undergoes small (10\%) oscillations in $a_{0}$ and $w_{0}$ [39]. In Fig. 4(b) the laser has further focused, compared to Fig. 4(a). The reduction of the laser pulse spot size leads to a shortening of the length of the bubble [40], characterized by the position behind the laser driver where the longitudinal electric field becomes positive. Some particles of the electron beam are then located in a defocusing phase, which corresponds to the rise of the electric field at the back of the bucket. This leads to truncation of the bunch, visible in the shorter scale length at the left-hand side of the bunch and the shorter overall bunch length in Fig. 4(c), as compared to Fig. 4(a).

Figure 5 shows the final trapped charge of the beam for a different channel matched radius. The final charge increases as the channel depth is relaxed. For a channel matched radius $w_{M}=9.2 \mu \mathrm{m}$, the final trapped charge is similar to the trapped charge without the plasma channel. However the final energy of the bunch is 2 times higher at dephasing than the unchanneled case, due to the fact that the laser pulse has not diffracted. Note that for $w_{M}=$ $9.2 \mu \mathrm{m}$ and $w_{M}=7.3 \mu \mathrm{m}$ the energy gain and energy spread are the same, whereas the emittance is $10 \%$ larger for $w_{M}=7.3 \mu \mathrm{m}$. At $w_{M}=6.5 \mu \mathrm{m}$ the energy gain is a third smaller for the same energy spread but with $10 \%$ lower emittance than for $w_{M}=9.2 \mu \mathrm{m}$. The purple square on Fig. 5 represents the final trapped charge, with a matched channel radius $w_{M}=9.2 \mu \mathrm{m}$, for the case where the laser pulse is focused at the point of the collision, i.e. inside the plasma, instead of at the entrance. Because this

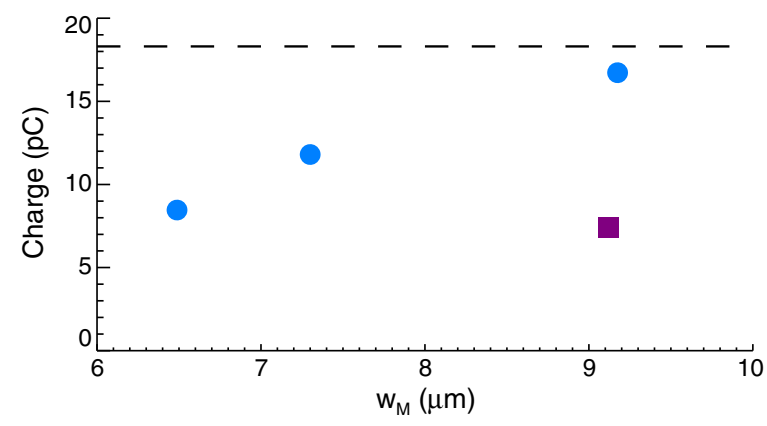

FIG. 5. Final trapped charge as a function of matched spot size $\left(w_{M}\right)$ for the plasma channel. Blue circles are for the laser driver focused at the entrance of the plasma while the purple square is for the laser driver focused at the point of collision. The dashed line represents the trapped charge without a plasma channel. focus position results in more severe variations during and after the interaction between the two laser pulses, less charge is trapped, showing the importance of controlling the focusing point of the laser beam, as well as the depth of the plasma channel.

Control of the focusing of the drive laser is critical to controlling the quality of the trapped electron beam. This was also noted in [27] where self-focusing of the laser affects the trapped charge. In the case of a laser under critical power for self-focusing, presence of the plasma channel, if not tuned properly, can result in a three-fold reduction in the charge of the trapped beam. However the channel is important to mitigate the diffraction of the laser pulse over the acceleration distance, and can in this case double the energy gain. With fine-tuning of the channel depth, one can keep the beam charge constant while obtaining higher energy gain.

\section{INJECTION WITHOUT PLASMA CHANNEL AND EXPERIMENTAL VALIDATION AND DESIGN}

To quantify the beam performance improvement offered by plasma channel guiding in the colliding pulse injector, simulations were performed for similar conditions without the presence of the plasma channel. In Sec. IV, it was shown that a correctly tuned plasma channel can double beam energy while achieving similar charge to an unchanneled configuration. Further simulations were conducted to parametrize the unchanneled injector performance as functions of timing, density, and collider amplitude. These parameters are chosen also to allow direct comparison with current experiments [22] run on the LBNL 10 TW laser. These and other experiments [16] are unchanneled to minimize complexity.

Variation of the delay between the two colliding laser pulses shows that electron trapping can be turned on and off. Trapping is turned on when the two pulses overlap within a $400 \mathrm{fs}$ window, and the charge is almost constant, with $\sim 20 \%$ variation, in the central 200 fs of this window. For the simulations we used the following laser parameters: $a_{0}=2, L_{0}=11.47 \mu \mathrm{m}, w_{0}=7.26 \mu \mathrm{m}$ and $a_{1}=0.5, L_{1}=$ $18 \mu \mathrm{m}, w_{1}=11 \mu \mathrm{m}$, and a plasma density of $n_{0}=$ $4.2 \times 10^{18} \mathrm{~cm}^{-3}$. The size of the window within which a significant amount of charge is trapped matches well between simulations and experiments [22], as shown in Fig. 6. Conditions for trapping depend solely on the spatial overlap of the two pulses, which is governed by pulse durations, spot sizes, and angle of the collision. Note that because the collision always occurs at the same location within the gas jet, the output energy will not depend on the delay between the two pulses: this is in contrast to a headon collision [16,27], where the delay between the pulses changes the acceleration distance and hence allows control of the output energy of the electron beam. 


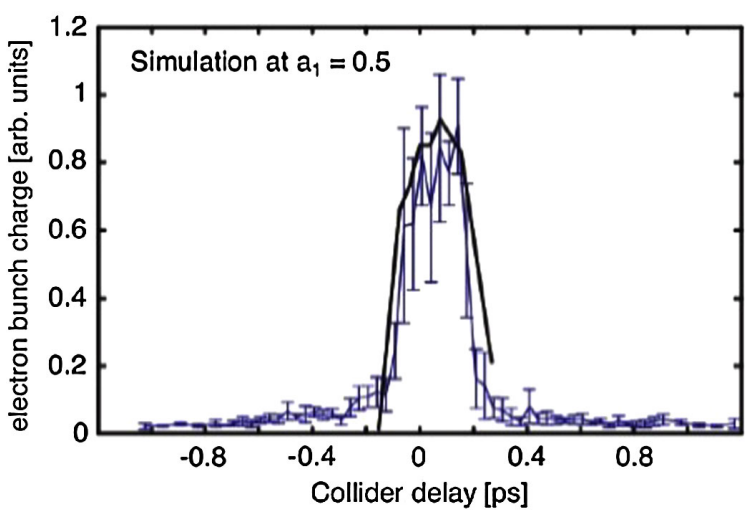

FIG. 6. Charge as a function of delay between the two colliding pulses for the experiments (blue) and the simulations (black). The experiments were operating with $a_{0} \sim 3$ and $a_{1} \sim 0.5$ [22].

Figure 7 shows the amount of trapped charge as a function of the plasma density for the same laser parameters. An optimum density of $n_{0} \sim 3 \times 10^{18} \mathrm{~cm}^{-3}$ for the trapped charge is observed for these parameters. Experiments show a similar optimum at $n_{0} \sim 4 \times 10^{18} \mathrm{~cm}^{-3}$, although with shorter driver pulse length. The density optimum is linked to the laser parameters used. Because laser parameters are kept constant, the volume of the beat wave stays the same, leading to fewer particles trapped as the density decreases compared to the optimum density. Also at lower density, the laser diffracts quickly in the absence of a plasma channel and self-guiding, reducing the wake amplitude. On the other hand, when the density increases the length of the driver pulses becomes long compared to the plasma wavelength $\lambda_{p}$, which reduces the wake amplitude, and hence increases the trapping threshold, leading to reduction of the trapped charge. This is consistent with having a higher optimum density with a shorter driver length in the experiments and in the case presented in Fig. 3. Note that this behavior is different from the case with the plasma channel, where controlled guiding of the laser pulse allows for a broader range of density where the trapped charge is high and stays approximately constant, allowing tuning of the beam energy independently of other parameters.

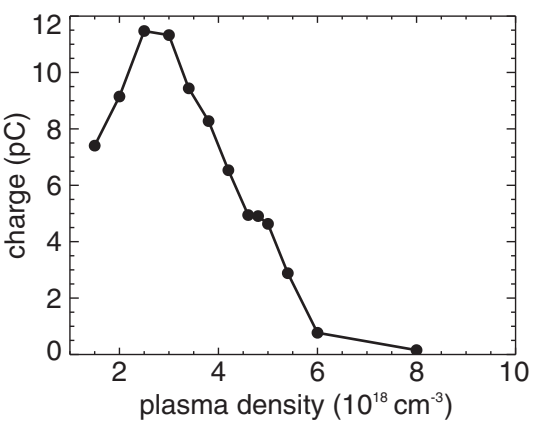

FIG. 7. Trapped charge as a function of the plasma density for laser parameters $a_{0}=2, L_{0}=11.47 \mu \mathrm{m}$ and $w_{0}=7.26 \mu \mathrm{m}$.

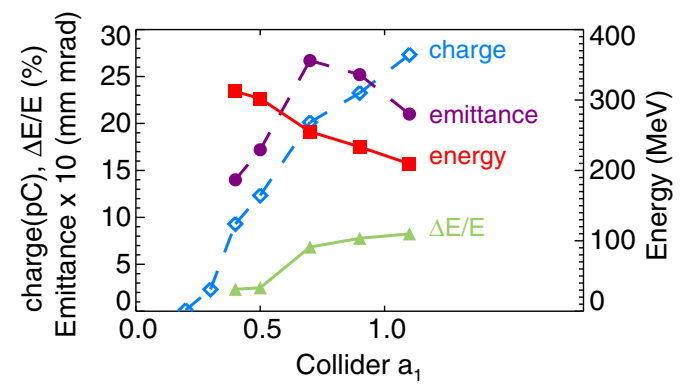

FIG. 8. Trapped charge (blue diamond), energy (red square), energy spread (green triangle), and transverse normalized emittance (purple circle) multiplied by 10 as a function of the collider laser intensity, for driver parameters $a_{0}=2, w_{0}=7.26 \mu \mathrm{m}$ and $L_{0}=11.47 \mu \mathrm{m}$, and plasma density $n_{0}=4.2 \times 10^{18} \mathrm{~cm}^{-3}$.

Test particle theory and simulations predict that the charge of the trapped bunch increases with the product of the two laser intensities $a_{0} a_{1}[15,17,24]$, which increases the overlap between the beat wave and the trapped separatrices. This is observed in Fig. 8 where the intensity of the collider pulse $a_{1}$ is varied for a constant density of $n_{0}=4.2 \times 10^{18} \mathrm{~cm}^{-3}$ and laser driver parameters described above. For these parameters we observe that trapping starts to occur for $a_{1}>0.2$. The amount of trapped charge then rapidly increases as $a_{1}$ increases, rolling off around $a_{1} \sim 1$. Similar behavior is observed in experiments $[22,26,41]$. The energy gain decreases and the energy spread and emittance increase as the charge increases. This is consistent with beam loading, as identified in previous colliding pulse studies [42]. Figure 9 shows the accelerating electric field in the case $a_{1}=0.4$ and $a_{1}=1.1$, as well as the longitudinal profile of the electron bunch. When $a_{1}$ is lower and less charge is trapped the longitudinal field amplitude is larger leading to higher energy gain. On the other hand, the wakefield created by the electron bunch leads to the creation of a strong gradient of the accelerating field inside the bunch. This gradient is lower when the charge is less, leading to less energy spread. Previous studies have shown that, by properly shaping the

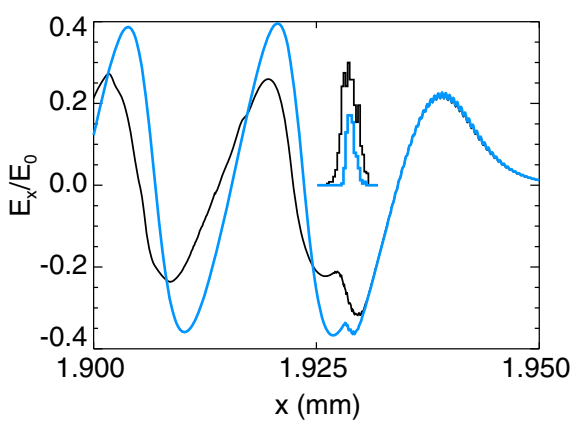

FIG. 9. Longitudinal electric field after injection of the electron bunch as a function of propagation distance for the parameters of Fig. 8 with $a_{1}=1.1$ (black) and $a_{1}=0.4$ (blue), and corresponding trapped bunch longitudinal profile. 
electron bunch, it is possible to achieve a constant accelerating field inside the bunch, leading to minimum energy spread $[35,43,44]$. In particular, increasing the length of the nearly Gaussian bunch would reduce the variation of the accelerating field inside the bunch. This should be achievable by changing parameters of the colliding laser pulses, or using the three-pulse scheme. This is part of further optimization and will not be studied here.

Injection in a uniform plasma profile offers the benefits of simplifying the experimental setup while obtaining high quality beams; in particular we have seen in Sec. IV that a plasma channel that is not tuned properly can be more detrimental to the beam properties. However, if the laser is below the critical power for self-focusing, one would achieve a more efficient acceleration with a plasma channel properly tuned. Also, as seen in Fig. 3, as opposed to Fig. 7, the plasma channel offers the opportunity to more independently tune the properties of the electron beam.

\section{EFFECTS OF NONGAUSSIAN LASER MODES ON INJECTION AND ACCELERATION}

High power lasers used to drive experiments often have non-Gaussian (higher order) mode components, and simulations have been used to evaluate the effect of not having a perfectly Gaussian laser pulse on the accelerated electron beam. The laser evolution is affected by the fact that higher order Gaussian modes propagate and self-focus differently. This can results in loss of self-focusing efficiency, modulation of the plasma wake, and steering of the electron beam off axis.

Laser pulse profiles obtained from experimental data are fitted with higher order Hermite-Gaussian modes up to third order (see Appendix B). Figures 10(a)-(c) show the transverse profile of different simulated laser pulses. Figure 10(a) is the reference fundamental Gaussian mode usually used in simulations. In Fig. 10(b) (pulse1) the fundamental Gaussian component is narrower but exhibits an asymmetric side lobe at $y \simeq 11 \mu \mathrm{m}$. In Fig. 10(c) (pulse2) the main fundamental Gaussian component has a larger spot size, resulting in a lower normalized intensity and two side lobes are located at $y \pm 42 \mu \mathrm{m}$. The energy of the laser pulse is kept constant in all cases. Other laser parameters are similar to those of Sec. V. The collider pulse is modeled as a perfect Gaussian with $a_{1}=0.5$, $L 1=18 \mu \mathrm{m}$, and $w_{1}=11 \mu \mathrm{m}$; the plasma density is uniform (no plasma channel) with $n_{0}=4.2 \times 10^{18} \mathrm{~cm}^{-3}$.

Figures 10(d)-(f) show the corresponding trapped electron beam properties at dephasing. The amount of trapped charge is proportional to the product of the two colliding laser intensities $a_{0} a_{1}$; hence the amount of trapped charge for pulse 1 is similar to the charge trapped with the perfect Gaussian mode. However, because pulse 2 has a lower intensity the amount of trapped charge is reduced by $60 \%$ and the quality of the beam is degraded. We note that with the perfect Gaussian mode the electron bunch is slightly
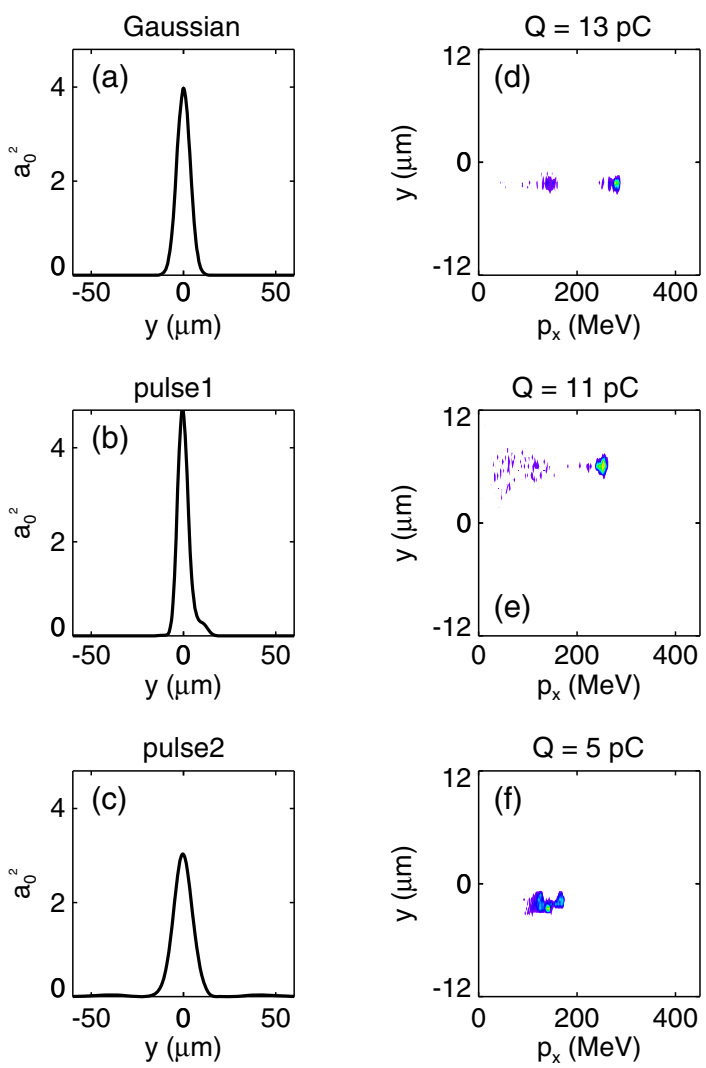

FIG. 10. Transverse laser profile for (a) the ideal Gaussian mode and (b),(c) combination of Hermite-Gaussian modes up to third order. The corresponding electron beam profile at dephasing is shown in (d)-(f).

steered off axis $(y \simeq-2.6 \mu \mathrm{m})$ [Fig. 10(d)]; this is due to the interaction of the driver with the collider, and we have observed that the offset of the bunch off axis can be controlled by controlling the timing between the two laser pulses. In the case of pulse 1 , the presence of a significant side lobe causes the laser intensity to peak off axis as the pulse propagates, leading to a steering of the electron beam off axis as seen in Fig. 10(e) where the beam is displaced by $y \simeq 7 \mu \mathrm{m}$ at dephasing.

Note that these simulations are performed without the plasma channel. In the case of pulse1, a plasma channel would cause the laser pulse intensity to oscillate around the plasma axis rather than continuously deviate from it [45]. This leads to oscillation of the back of the bubble and loss of charge, as some particles initially trapped end up in a defocusing region due to the laser oscillations. Such a case is shown in Fig. 11, which shows the laser transverse intensity profile as a function of propagation distance in the plasma channel, and the corresponding amount of charge trapped inside the bubble, after the interaction has occurred. After the first oscillation of the laser pulse in the channel about half of the initial trapped charge is lost due to oscillations of the back of the bubble. The rest of the charge stays trapped even though the laser continues to oscillate. However, the laser oscillation 


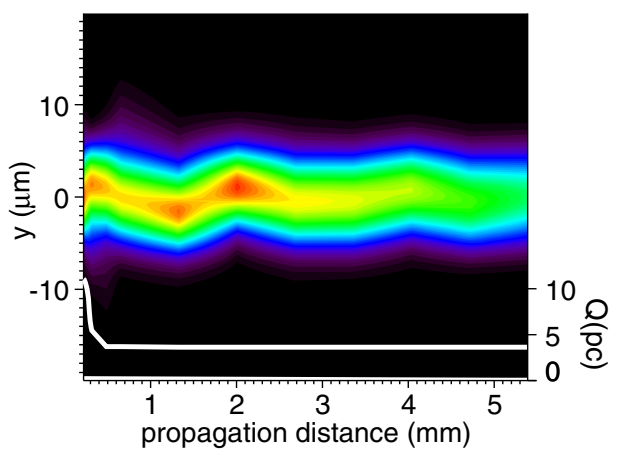

FIG. 11. Laser transverse intensity profile as a function of propagation distance for pulse 1 in a plasma channel with matched spot $w_{M}=9.2 \mu \mathrm{m}$. The white line represents the trapped charge (right $y$ axis).

will cause the electron beam to oscillate as well around the axis, leading to steering of the beam off axis. These simulations illustrate the importance of reducing asymmetric mode components for both unchanneled and channeled cases. The results derived here from 2D simulations should be regarded as a lower limit, as focusing and related effects typically become more pronounced in 3D (representative of experiments).

\section{CONCLUSION}

Colliding pulse triggered injection is one method being explored to produce reliable and tunable high quality electron beams in LPA. Here, we performed self-consistent, particle-in-cell simulations to predict and design CPI experiments currently taking place at LBNL. So far, experimental results have shown good agreement with simulation for timing, density and collider intensity scans, which gives confidence in the ability of the simulations to predict the outcome of the experiments. To further understand how to optimize the experimental results we have studied the impact of non-Gaussian laser modes and the importance of laser evolution. Tunability and scalability of the accelerator was also demonstrated.

We showed that it is possible to control the properties of the trapped electron beam as follows: (i) the trapping can be turned on and off by controlling the overlap of the two laser pulses, (ii) the charge in the electron beam can be increased by increasing the intensity of the collider pulse, consistent with test particle simulations $[17,18]$, (iii) in the presence of a plasma channel, the final energy of the electron beam can be controlled independently of other beam parameters by changing the dephasing length, i.e., the plasma density, such that $E \sim 1 / \sqrt{n_{0}}$, somewhat similarly to [27] where the final energy of the beam is controlled by changing the injection point inside the plasma, hence changing the acceleration distance, (iv) final properties of the beam can be designed starting from the beam properties for a given set of parameters and using linear scaling laws. For the parameters accessible in current LBNL experiments, we showed that a $20 \mathrm{pC}$ electron beam can be accelerated to $300 \mathrm{MeV}$ with $2 \%$ energy spread and a transverse normalized emittance of order $1 \mathrm{~mm}$ mrad using a $10 \mathrm{TW}$ laser system, which would otherwise require a $30 \mathrm{TW}$ laser system when relying on self-injection and self-guiding.

Simulations enabled access to the internal dynamics of the trapping and acceleration process and showed the importance of controlling the shape of both the plasma channel and the laser mode profile to obtain the highest possible quality beam. Modification of the wake due to laser pulse evolution can lead to loss of a portion of the charge initially trapped. The quality and tunability of the electron beam can be improved by using a third laser pulse. This allows the injection process to be completely decoupled from the acceleration. This will be the subject of future study in both simulations and experiments.

\section{ACKNOWLEDGMENTS}

The authors would like to thank E. Esarey and C. B. Schroeder for useful discussions. This work was supported by the Department of Energy, Office of National Nuclear Security Administration, NA-22, and Office of Science, Office of High Energy Physics under Contract No. DEAC02-05CH11231, and via the ComPASS SciDAC project, Grants No. DE-FC02-07ER41499 and No. DESC0008920, and by Tech-X Corporation. This work used resources of the National Energy Research Scientific Computing Center, which is supported by the Office of Science of the U.S. Department of Energy under Contract No. DE-AC02-05CH11231. We acknowledge the VORPAL development team: D. Alexander, K. Amyx, E. Angle, T. Austin, G. I. Bell, D. L. Bruhwiler, J. R. Cary, E. CormierMichel, B. M. Cowan, R. K. Crockett, Y. Choi, D. A. Dimitrov, M. Durant, B. Jamroz, M. Koch, S. E. Kruger, A. Likhanskii, M.C. Lin, M. Loh, J. Loverich, S. Mahalingam, P. J. Mullowney, C. Nieter, K. Paul, I. Pogorelov, C. Roark, D. Robertson, B. T. Schwartz, S. W. Sides, D. N. Smithe, P. H. Stoltz, S. A. Veitzer, D. J. Wade-Stein, G. R. Werner, N. Xiang, and C. D. Zhou.

\section{APPENDIX A: CORRECT LAUNCHING OF A LASER PULSE IN A PIC SIMULATION}

To verify the correct form of the laser pulses in our simulations we compared the fields generated in our simulation with a well-known analytical approximation. We verified the error convergence for our simulations with increasing resolution to the analytical values accounting for the approximations in the analytical form.

Since no exact analytical form for a Gaussian laser pulse exists, an approximation is used that works well in the paraxial limit. In the paraxial approximation, the vector potential is written in the following form:

$$
A_{j}(\vec{r}, t)=\psi(r, t) g(\phi) \exp (i \phi)
$$


where $j$ is the Cartesian component, $\psi$ is the spatial envelope, $r_{\perp}=\left(x^{2}+y^{2}\right), g$ is the temporal envelope, and $\phi=k z-\omega t$ is the phase. This form represents a solution to

$$
\Delta^{2} \psi+2 i k \frac{\partial \psi}{\partial z}\left(1-i g^{\prime} / g\right)=0,
$$

where it is assumed that $\left|g^{\prime} / g\right| \ll 1$, meaning the envelope is slowly varying. Depending on the functional form of $g(\phi)$, this ratio can become large at the edges of the laser pulse, where $g$ vanishes.

Because of the approximate nature of the solution, errors due to finite-difference time-domain simulations with a code like VORPAL will not converge to zero when compared to standard analytic formulas; rather, one expects to see second-order convergence to a finite offset from zero. An example is shown in Fig. 12. The magnitude of this offset will itself go to zero as the temporal envelope is increased, resulting in smaller derivatives and, hence, greater accuracy of the paraxial approximation.

There are other assumptions built into the paraxial approximation, but the requirement of small envelope derivatives appears to be the dominant contributor to the residual term shown in Fig. 12. In Fig. 13, it is shown how the residual decreases as a laser envelope of fixed shape is made longer with respect to the laser wavelength.

The analysis discussed above is for a laser pulse launched along the longitudinal axis from the center of left domain boundary, as is typically done in laser-plasma simulations for the drive pulse. For colliding pulse simulations, it is also necessary to launch a second laser pulse from the right side of the domain, with a specified angle of propagation. Hence, the analysis above was generalized for this case. Analogous to Fig. 12, we show in Fig. 14 how the simulated laser pulse converges to the analytic form as the mesh resolution is improved. The size

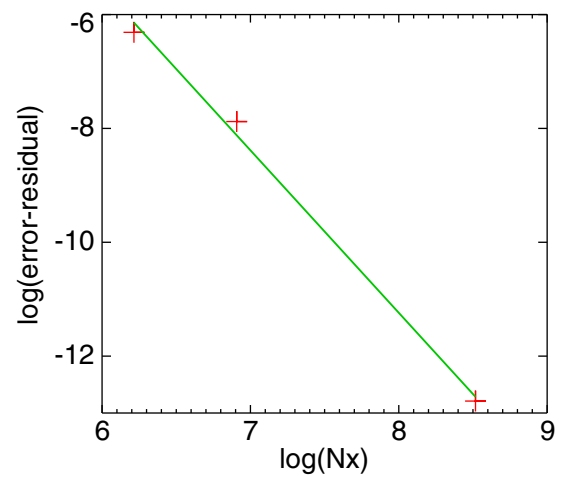

FIG. 12. Results from VORPAL simulations, in which a laser pulse with the standard paraxial form is launched from one surface of the simulation domain, and then compared with the analytic form after it has propagated onto the mesh. In order to see numerical convergence with resolution, it is required that one subtract from the error a residual constant that depends upon details of the laser pulse length and shape. In this example, the convergence is faster than quadratic, which cannot be expected in general.

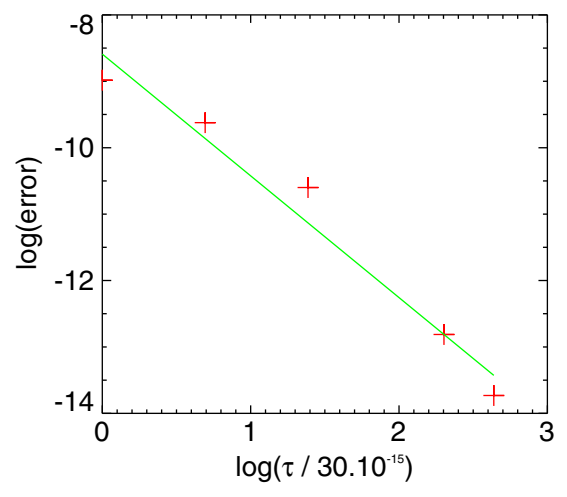

FIG. 13. The magnitude of the residual factor (discussed above and in Fig. 12), which is seen in convergence studies of a laser pulse launched from the simulation boundary, is shown here to decrease quadratically with the length of the laser pulse envelope, characterized by the pulse length $\tau$ here given in seconds (for fixed shape and fixed laser wavelength).

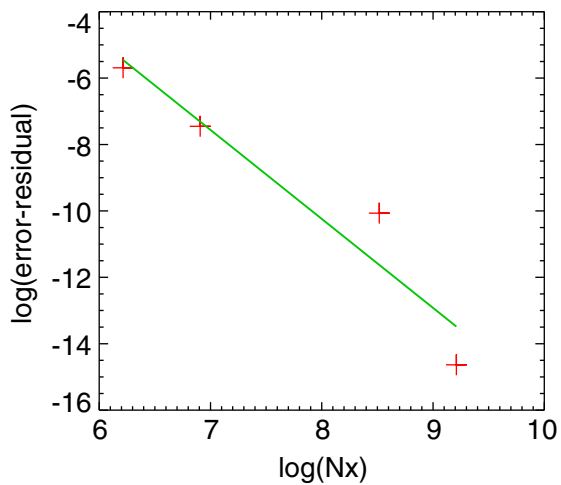

FIG. 14. Analogous to Fig. 12 above, except here the paraxial laser pulse was launched at an angle from the right-hand surface of the simulation domain, and then compared with the analytic form after it has propagated onto the mesh. As before, it is required that one subtract from the error a residual constant that depends upon details of the laser pulse length and shape.

of the residual factor in Fig. 14 is reduced for longer laser pulses; however, we do not present a figure as it is very similar to Fig. 13.

We verified that a laser pulse launched either from the left side of the box, as is typical in laser plasma simulations, or the right side of the box with an angle is close to the analytical expression, the error converging quadratically with resolution. Typical resolution used in simulations yields an error of the percent level, which is acceptable.

\section{APPENDIX B: FITTING AN EXPERIMENTAL MODE PROFILE WITH HIGHER ORDER MODES}

To simulate the effects of non-Gaussian laser modes present in experiments, we numerically fitted the measured data to a combination of several Hermite-Gaussian modes. We parametrized the laser pulse as shown in Eq. (B1): 


$$
\begin{aligned}
E(x, y)= & E 0_{1} \exp \left(-\frac{\left(x-x_{\text {focus }_{1}}\right)^{2}}{w_{x_{1}}^{2}}-\frac{\left(y-y_{\text {focus }_{1}}\right)^{2}}{w_{y_{1}}^{2}}\right)+E 0_{2} \exp \left(-\frac{\left(x-x_{\text {focus }_{2}}\right)^{2}}{w_{x_{2}}^{2}}-\frac{\left(y-y_{\text {focus }_{2}}\right)^{2}}{w_{y_{2}}^{2}}\right) \\
& +E 0_{3} \exp \left(-\frac{\left(x-x_{\text {focus }_{3}}\right)^{2}}{w_{x_{3}}^{2}}-\frac{\left(y-y_{\text {focus }_{3}}\right)^{2}}{w_{y_{3}}^{2}}\right)\left(a_{1} \frac{\operatorname{herm}_{1}\left[\sqrt{2}\left(x-x_{\text {focus }_{3}}\right) / w_{x_{3}}\right]}{\sqrt{2}} \frac{\operatorname{herm}_{1}\left[\sqrt{2}\left(y-y_{\text {focus }_{3}}\right) / w_{y_{3}}\right]}{\sqrt{2}}\right. \\
& +a_{2} \frac{\operatorname{herm}_{2}\left[\sqrt{2}\left(x-x_{\text {focus }_{3}}\right) / w_{x_{3}}\right]}{\sqrt{22^{2}}} \frac{\operatorname{herm}_{1}\left[\sqrt{2}\left(y-y_{\text {focus }_{3}}\right) / w_{y_{3}}\right]}{\sqrt{22^{2}}} \\
& \left.+a_{3} \frac{\operatorname{herm}_{3}\left[\sqrt{2}\left(x-x_{\text {focus }_{3}}\right) / w_{x_{3}}\right]}{\sqrt{62^{3}}} \frac{\operatorname{herm}_{1}\left[\sqrt{2}\left(y-y_{\text {focus }_{3}}\right) / w_{y_{3}}\right]}{\sqrt{62^{3}}}\right),
\end{aligned}
$$

$$
I(x, y)=E(x, y)^{2} .
$$

Here $E 0_{i}$ are the amplitudes for each pulse, $w_{x_{i}}$ and $w_{y_{i}}$ are the transverse widths and $x_{\text {focus }_{i}}$ and $y_{\text {focus }_{i}}$ are the transverse focal points for the $i$ th pulse. Also $a_{m}$ are the amplitudes for each Hermite polynomial. In Figs. 15 and 16 we show our fit to focal-plane intensity data taken in 2009 and 2003. These were fit using the
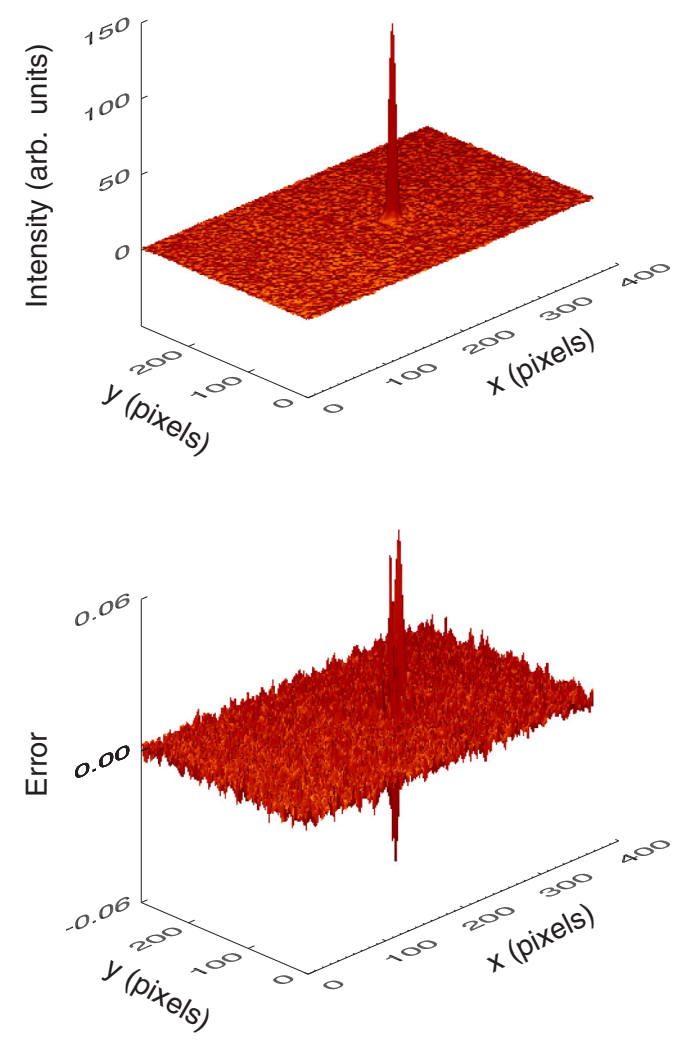

FIG. 15. Measured intensity data taken in 2003 and the residual using error $=($ measured data - fitdata $) / \max [\operatorname{abs}($ measured data $)]$. The calibration is $0.9 \mu \mathrm{m}$ per pixel in the $x$ directions and $1.057 \mu \mathrm{m}$ per pixel in the $y$ direction. The error between measured and fitted data is less than $6 \%$.
Levenberg-Marquardt least-squares fit with the Interactive Data Language fitting function MPFITFUN [46]. Here the cross terms of $i \theta$ in the Hermite expansion and the oscillatory terms in the regular Gaussian expression produced in Eq. (B2) have been ignored. This would have expanded the fitted parameter space too significantly without corresponding phase data to fit. The error between the original data and the fit stays below $6 \%$ in the region of interest.
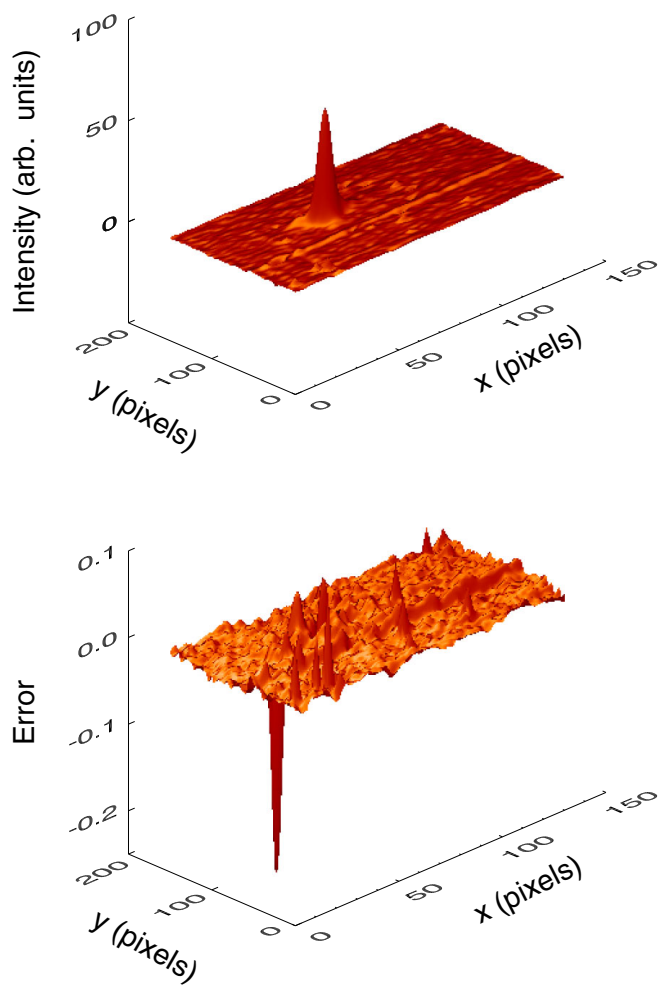

FIG. 16. Measured intensity data taken in 2009 and the residual error. The calibration is $0.9 \mu \mathrm{m}$ per pixel in the $x$ directions and $1.057 \mu \mathrm{m}$ per pixel in the $y$ direction. The error has a single $\sim 22 \%$ spike outside of the interaction region but the rest is well below $5 \%$. 
TABLE I. Mean energy, relative rms energy spread, charge and normalized transverse emittance of the trapped beam at dephasing as a function of longitudinal grid resolution, for cubic (third order) interpolation.

\begin{tabular}{lcccc}
\hline \hline & $\begin{array}{c}\langle E\rangle \\
(\mathrm{MeV})\end{array}$ & $\begin{array}{c}\Delta E / E \mathrm{rms} \\
(\%)\end{array}$ & $Q(p C)$ & $\begin{array}{c}\epsilon_{n} \\
(\mathrm{~mm} \text { mrad })\end{array}$ \\
\hline$d x=\lambda_{0} / 24$ & 390 & 2.0 & 15 & 6.1 \\
$d x=\lambda_{0} / 36$ & 363 & 2.3 & 20 & 2.2 \\
$d x=\lambda_{0} / 48$ & 367 & 2.3 & 20 & 1.4 \\
$d x=\lambda_{0} / 60$ & 360 & 2.3 & 20 & 1.1 \\
\hline \hline
\end{tabular}

TABLE II. Mean energy, relative rms energy spread, charge, and normalized transverse emittance of the trapped beam at dephasing as a function of interpolation order, for $d x=\lambda_{0} / 36$.

\begin{tabular}{lcccc}
\hline \hline & $\begin{array}{l}\langle E\rangle \\
(\mathrm{MeV})\end{array}$ & $\begin{array}{c}\Delta E / E \text { rms } \\
(\%)\end{array}$ & $Q(p C)$ & $\begin{array}{c}\epsilon_{n} \\
(\mathrm{~mm} \mathrm{mrad})\end{array}$ \\
\hline 1st order & 362 & 2.6 & 20 & 2.4 \\
2nd order & 362 & 2.4 & 20 & 2.3 \\
3rd order & 363 & 2.3 & 20 & 2.2 \\
4th order & 364 & 2.4 & 20 & 1.8 \\
\hline \hline
\end{tabular}

\section{APPENDIX C: NUMERICAL CONVERGENCE STUDY}

Previous studies have shown that LPA PIC simulation results can be sensitive to numerical parameters [47]. Here, we study the properties of the trapped electron beam at dephasing as a function of resolution and method of interpolation, for the colliding pulse simulation parameters of the present study.

Table I shows the beam properties for different resolutions, using the third order spline, or cubic, interpolation to obtain the fields at the particle position and to deposit the particle current on the grid [48]. The results are converged for $d x \gtrsim \lambda_{0} / 36$, except for the emittance, which requires extremely high resolution to achieve convergence. Table II shows that the interpolation order does not significantly change the results, at the resolution of $d x=\lambda_{0} / 36$. This is the resolution used for parameter scans in this study.

[1] E. Esarey, C. B. Schroeder, and W. P. Leemans, Rev. Mod. Phys. 81, 1229 (2009).

[2] J. Faure, Y. Glinec, A. Pukhov, S. Kiselev, S. Gordienko, E. Lefebvre, J.-P. Rousseau, F. Burgy, and V. Malka, Nature (London) 431, 541 (2004).

[3] C. G. R. Geddes, C. Toth, J. van Tilborg, E. Esarey, C. B. Schroeder, D. L. Bruhwiler, C. Nieter, J. Cary, and W. P. Leemans, Nature (London) 431, 538 (2004).

[4] S. P. D. Mangles, C. D. Murphy, Z. Najmudin, A. G. R. Thomas, J. L. Collier, A. E. Dangor, E. J. Divall, P. S. Foster, J. G. Gallacher, C. J. Hooker, D. A. Jaroszynski, A. J. Langley, W. B. Mori, P. A. Norreys, F. S. Tsung, R.
Viskup, B. R. Walton, and K. Krushelnick, Nature (London) 431, 535 (2004).

[5] W. P. Leemans, B. Nagler, A. J. Gonsalves, C. Tóth, K. Nakamura, C. G. R. Geddes, E. Esarey, C. B. Schroeder, and S. M. Hooker, Nat. Phys. 2, 696 (2006).

[6] J. Osterhoff, A. Popp, Z. Major, B. Marx, T. P. Rowlands-Rees, M. Fuchs, M. Geissler, R. Hörlein, B. Hidding, S. Becker, E. A. Peralta, U. Schramm, F. Grüner, D. Habs, F. Krausz, S. M. Hooker, and S. Karsch, Phys. Rev. Lett. 101, 085002 (2008).

[7] C. G. R. Geddes, K. Nakamura, G. R. Plateau, C. Toth, E. Cormier-Michel, E. Esarey, C. B. Schroeder, J. R. Cary, and W. P. Leemans, Phys. Rev. Lett. 100, 215004 (2008).

[8] A. J. Gonsalves, K. Nakamura, C. Lin, D. Panasenko, S. Shiraishi, T. Sokollik, C. Benedetti, C. B. Schroeder, C. G. R. Geddes, J. van Tilborg, J. Osterhoff, E. Esarey, C. Toth, and W. P. Leemans, Nat. Phys. 7, 862 (2011).

[9] M. Chen, Z.-M. Sheng, Y.-Y. Ma, and J. Zhang, J. Appl. Phys. 99, 056109 (2006).

[10] M. Chen, E. Esarey, C. B. Schroeder, C. G. R. Geddes, and W. P. Leemans, Phys. Plasmas 19, 033101 (2012).

[11] A. Pak, K. A. Marsh, S. F. Martins, W. Lu, W. B. Mori, and C. Joshi, Phys. Rev. Lett. 104, 025003 (2010).

[12] C. McGuffey, A. G. R. Thomas, W. Schumaker, T. Matsuoka, V. Chvykov, F. J. Dollar, G. Kalintchenko, V. Yanovsky, A. Maksimchuk, K. Krushelnick, V. Y. Bychenkov, I. V. Glazyrin, and A. V. Karpeev, Phys. Rev. Lett. 104, 025004 (2010).

[13] M. Chen, E. Esarey, C. G. R. Geddes, E. Cormier-Michel, C. B. Schroeder, S. S. Bulanov, C. Benedetti, L. L. Yu, S. Rykovanov, D. L. Bruhwiler, and W. P. Leemans, Phys. Rev. ST Accel. Beams 17, 051303 (2014).

[14] B. B. Pollock, C. E. Clayton, J. E. Ralph, F. Albert, A. Davidson, L. Divol, C. Filip, S. H. Glenzer, K. Herpoldt, W. Lu, K. A. Marsh, J. Meinecke, W. B. Mori, A. Pak, T. C. Rensink, J. S. Ross, J. Shaw, G. R. Tynan, C. Joshi, and D. H. Froula, Phys. Rev. Lett. 107, 045001 (2011).

[15] E. Esarey, R. F. Hubbard, W. P. Leemans, A. Ting, and P. Sprangle, Phys. Rev. Lett. 79, 2682 (1997).

[16] J. Faure, C. Rechatin, A. Norlin, A. Lifschitz, Y. Glinec, and V. Malka, Nature (London) 444, 737 (2006).

[17] C. B. Schroeder, P. B. Lee, J. S. Wurtele, E. Esarey, and W. P. Leemans, Phys. Rev. E 59, 6037 (1999).

[18] G. Fubiani, E. Esarey, C. B. Schroeder, and W. P. Leemans, Phys. Rev. E 70, 016402 (2004).

[19] J. R. Cary, R. E. Giacone, C. Nieter, and D. L. Bruhwiler, Phys. Plasmas 12, 056704 (2005).

[20] H. Kotaki, I. Daito, M. Kando, Y. Hayashi, K. Kawase, T. Kameshima, Y. Fukuda, T. Homma, J. Ma, L.-M. Chen, T. Z. Esirkepov, A. S. Pirozhkov, J. K. Koga, A. Faenov, T. Pikuz, H. Kiriyama, H. Okada, T. Shimomura, Y. Nakai, M. Tanoue, H. Sasao, D. Wakai, H. Matsuura, S. Kondo, S. Kanazawa, A. Sugiyama, H. Daido, and S. V. Bulanov, Phys. Rev. Lett. 103, 194803 (2009).

[21] C. Toth, E. Esarey, C. Geddes, W. Leemans, K. Nakamura, D. Panasenko, C. Schroeder, D. Bruhwiler, and J. Cary, in Proceedings of the 22nd Particle Accelerator Conference, PAC-2007, Albuquerque, NM (IEEE, New York, 2007), pp. 2975-2977. 
[22] G. R. Plateau, C. G. R. Geddes, N. H. Matlis, E. CormierMichel, D. E. Mittelberger, K. Nakamura, C. B. Schroeder, E. Esarey, and W. P. Leemans, AIP Conf. Proc. 1299, 180 (2010).

[23] TT-MOBILE, High peak power lasers, amplitude technologies, http://www.amplitude-technologies.com//?fond= produit\&id_produit=11\&id_rubrique $=1$.

[24] C. Rechatin, J. Faure, A. Lifschitz, V. Malka, and E. Lefebvre, Phys. Plasmas 14, 060702 (2007).

[25] Z. Sheng, W. Wang, R. Trines, P. Norreys, M. Chen, and J. Zhang, Eur. Phys. J. Spec. Top. 175, 49 (2009).

[26] C. Rechatin, J. Faure, A. Ben-Ismail, J. Lim, R. Fitour, A. Specka, H. Videau, A. Tafzi, F. Burgy, and V. Malka, Phys. Rev. Lett. 102, 164801 (2009).

[27] X. Davoine, E. Lefebvre, J. Faure, C. Rechatin, A. Lifschitz, and V. Malka, Phys. Plasmas 15, 113102 (2008).

[28] X. Davoine, E. Lefebvre, C. Rechatin, J. Faure, and V. Malka, Phys. Rev. Lett. 102, 065001 (2009).

[29] C. Rechatin, J. Faure, A. Lifschitz, X. Davoine, E. Lefebre, and V. Malka, New J. Phys. 11, 013011 (2009).

[30] C. Nieter and J. R. Cary, J. Comp. Physiol. 196, 448 (2004).

[31] C. G. R. Geddes, D. L. Bruhwiler, J. R. Cary, E. H. Esarey, A. J. Gonsalves, C. Lin, E. Cormier-Michel, N. H. Matlis, K. Nakamura, M. Bakeman, D. Panasenko, G. R. Plateau, C. B. Schroeder, C. Toth, and W. P. Leemans, AIP Conf. Proc. 1099, 666 (2009).

[32] C. G. R. Geddes et al., SciDAC Rev. 13, 13 (2009).

[33] E. Cormier-Michel, V. H. Ranjbar, D. L. Bruhwiler, M. Chen, C. G. R. Geddes, E. Esarey, C. B. Schroeder, and W. P. Leemans, AIP Conf. Proc. 1299, 215 (2010).

[34] C. G. R. Geddes, C. Toth, J. van Tilborg, E. Esarey, C. B. Schroeder, J. Cary, and W. P. Leemans, Phys. Rev. Lett. 95, 145002 (2005).

[35] E. Cormier-Michel, C. G. R. Geddes, E. Esarey, C. B. Schroeder, D. L. Bruhwiler, K. Paul, B. Cowan, and W. P. Leemans, AIP Conf. Proc. 1086, 297 (2009).
[36] K. Németh, B. Shen, Y. Li, H. Shang, R. Crowell, K. C. Harkay, and J. R. Cary, Phys. Rev. Lett. 100, 095002 (2008).

[37] J.-L. Vay, C. G. R. Geddes, E. Esarey, C. B. Schroeder, W. P. Leemans, E. Cormier-Michel, and D. P. Grote, Phys. Plasmas 18, 123103 (2011).

[38] S. Kalmykov, S. A. Yi, V. Khudik, and G. Shvets, Phys. Rev. Lett. 103, 135004 (2009).

[39] C. Benedetti, C. B. Schroeder, E. Esarey, and W. P. Leemans, Phys. Plasmas 19, 053101 (2012).

[40] S. Y. Kalmykov, A. Beck, S. A. Yi, V. N. Khudik, M. C. Downer, E. Lefebvre, B. A. Shadwick, and D. P. Umstadter, Phys. Plasmas 18, 056704 (2011).

[41] N. H. Matlis, C. G. R. Geddes, G. R. Plateau, E. Esarey, C. Schroeder, D. Bruhwiler, E. Cormier-Michel, M. Chen, L. Yu, and W. P. Leemans, AIP Conf. Proc. 1507, 717 (2012).

[42] C. Rechatin, X. Davoine, A. Lifschitz, A. B. Ismail, J. Lim, E. Lefebvre, J. Faure, and V. Malka, Phys. Rev. Lett. 103, 194804 (2009).

[43] T. Katsouleas, S. Wilks, P. Chen, J. Dawson, and J. Su, Part. Accel. 22, 81 (1987).

[44] M. Tzoufras, W. Lu, F. S. Tsung, C. Huang, W. B. Mori, T. Katsouleas, J. Vieira, R. A. Fonseca, and L. O. Silva, Phys. Plasmas 16, 056705 (2009).

[45] A. J. Gonsalves, K. Nakamura, C. Lin, J. Osterhoff, S. Shiraishi, C. B. Schroeder, C. G. R. Geddes, C. Toth, E. Esarey, and W. P. Leemans, Phys. Plasmas 17, 056706 (2010).

[46] IDL utilities made freely available to the general public, http://cow.physics.wisc.edu/ craigm/idl/idl.html.

[47] E. Cormier-Michel, B. A. Shadwick, C. G. R. Geddes, E. Esarey, C. B. Schroeder, and W. P. Leemans, Phys. Rev. E 78, 016404 (2008).

[48] T. Esirkepov, Comput. Phys. Commun. 135, 144 (2001). 